\title{
Spectroscopy of Blue Compact Galaxies *
}

\author{
A.A.M. Esposito and P. Rafanelli, Padova, Italy \\ University of Padova, Astronomy Department \\ V.A. Lipovetski†, Selenchuk, Russia \\ Special Astrophysical Observatory
}

Received 1997 May 24; accepted 1997 July 2

Blue Compact Galaxies (BCGs) are gas-rich systems undergoing very massive starburst events which involve the entire galaxy. We performed a spectroscopic study of 12 BCG in order to determine metallicities and helium content. Some objects show presence of broadened emission lines, probably due to internal motion of the interstellar medium, caused by intense stellar winds and supernova explosions.

Key words: galaxies: abundances - compact galaxies - ISM - starbursts - HII regions

AAA subject classification: $132,152,158$

1.

\section{Introduction}

Blue Compact Galaxies (BCGs) are gas-rich systems undergoing a very massive burst of star formation. Since their discovery in 1970 by Sargent and Searle, they were called isolated extragalactic HII regions, because their spectra resemble those of giant HII regions such as 30 Dor in the LMC. Today, the most widely accepted denomination is "HII galaxies", because their appearence is completely dominated by the HII region at their center. In almost all cases the HII region is superimposed to an underlying dwarf galaxy, and this is the reason why we call them BCGs. Their appearence on Palomar photographic plates is quite always stellar-like as size, with a fuzzy structure and no dominant nucleus (see Fig. a) but there are remarkable exceptions (Fig. b).

It is generally believed that BCGs are very young objects, due to their high content of neutral hydrogen, with gas fraction $\sim 0.1 \div 0.5$ of the total mass, and very low metallicities (from $1 / 3$ to $1 / 70$ of the solar value). For this reason these galaxies are claimed to be the best laboratory for the study of primordial helium content $\left(\mathrm{Y}_{p}\right)$ via the determination of the oxygen content (see Campbell (1992) for a review). The knowledge of $Y_{p}$ is crucial in verifying predictions of the standard big bang nucleosynthesis theory, in particular the baryon-to-photon ratio, and the lifetime of the neutron $\tau_{\nu}$ (Pagel et al. 1992). We performed high resolution spectral analysis of 12 BCGs, in order to determine their metallicities and helium content. In Section 2 we present the sample of galaxies under study, in Section 3 we describe the data reduction. Several objects of the sample present evidence of broadening of emission lines, and this will be discussed in details in Section 4. Finally, a summary of the results is presented in Section 5 .

2. The sample

A substantial part of blue emission line objects found in objective prism surveys for quasar search (e.g. UM, Case, FBS, SBS, HES) and in surveys for galaxies with emission lines ( $\mathrm{Zw}$ and Mrk) is formed by BCGs. The galaxies we studied are 12 metal-deficient objects chosen from a complete sample of emission line galaxies assembled from an objective prism survey carried out with the $1 \mathrm{~m}$ ESO Schmidt telescope as a key project "Hamburg/ESO Survey for bright QSOs" (HES). With a magnitude limit $m_{v} \sim 18$, the HES provides a well-defined sample of low luminosity

"Poster presented at the Asiago Meeting "Environmental and physical properties of nearby AGN" on 23-24 April, 1997 

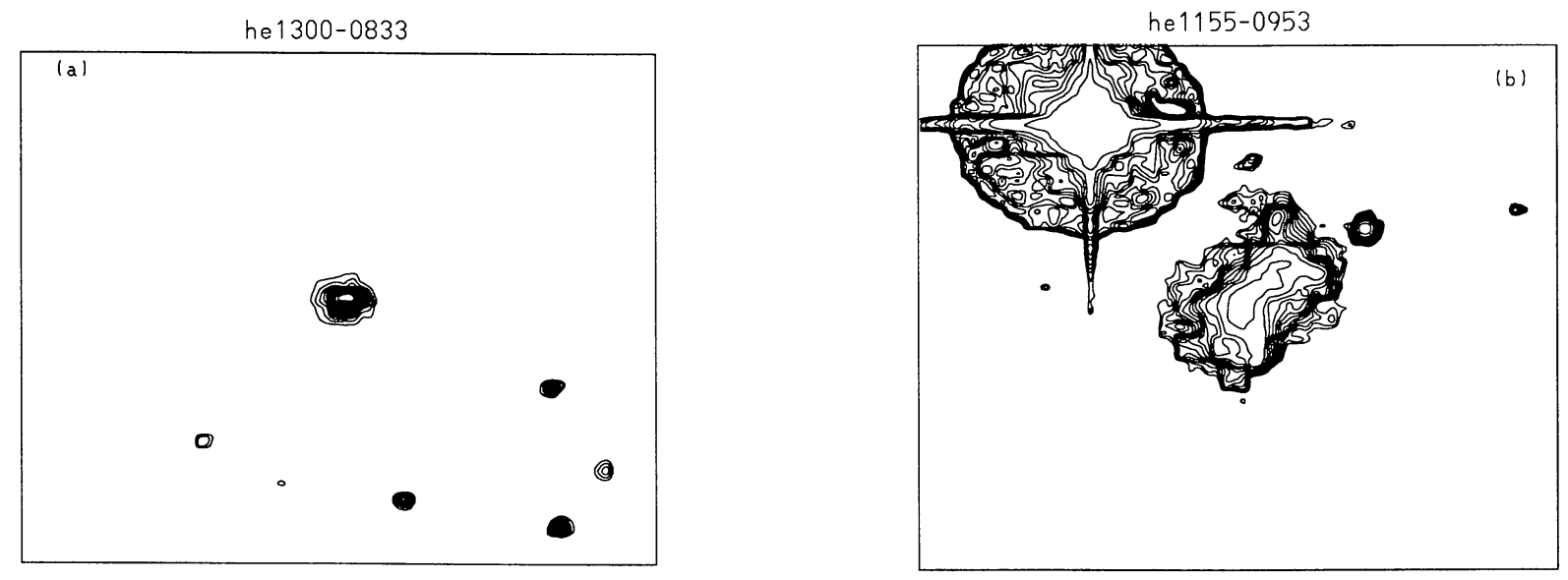

Fig. 1. (a) Contour map of he1300-0833; the other objects in the field are stars. (b) Countour map of he1155-0953: note the four brilliant knots of emission (see also Fig. 3[up]). Images are taken from the uncompressed Digitized Sky Survey through SkyView; square sides are roughly 3 arcmin.

galaxies with high-excitation spectra and strong emission lines in a sky region defined by $10^{\mathrm{h}} \leq \alpha \leq 13^{\mathrm{h}} 03^{\mathrm{m}}$ and $-15^{\circ} \leq \delta \leq-5^{\circ}$.

All objects whose low dispersion spectra on the objective prism plates showed strong emission lines and a weak continuum were classified using short exposure spectra taken with the 1.52-mESO telescope. This follow-up spectroscopic work allowed to define a complete sample of $\sim 20$ BCGs (other emission-line objects such as Seyfert galaxies, massive starburst galaxies or quasars being removed).

The subsequent spectroscopy we carried out in this work will allow us to give accurate abundances of $\mathrm{O}, \mathrm{N}$ and $\mathrm{S}$. The completion of this program can increase the number of galaxies with well measured He abundances, and provide crucial data for testing modern cosmological models for the standard Big Bang nucleosynthesis.

Campbell (1992), analyzing all avaliable data of BCGs, found a large physical dispersion in the $\mathrm{O} / \mathrm{H}-\mathrm{He} / \mathrm{H}$ relation used to infer the abundance of primordial helium $\left(Y_{p}\right)$. She requested more high-quality data for BCGs in the range $7.0 \leq 12+\log (\mathrm{O} / \mathrm{H}) \leq 8.0$. The metallicity of the sample spans from $1 / 5$ to $1 / 20$ solar, and this locates our BCGs in the gap cited by Campbell.

The most extensive survey until now is that of Campbell et al. (1984; 32 objects). There are also 12 objects studied by Kunth \& Sargent (1983). Izotov and collaborators $(1994 ; 1996)$ have studied $10+3$ BCGs.

The object under study are those listed in Table 1.

Table 1. The sample of BCGs

\begin{tabular}{|c|c|c|c|c|c|c|c|c|c|c|}
\hline Object & \multicolumn{3}{|c|}{ R.A. } & \multicolumn{3}{|c|}{ Dec } & Date & $\begin{array}{c}\text { Observing } \\
\text { time }\end{array}$ & $\begin{array}{c}\text { Slit } \\
\text { width }\end{array}$ & P.A. \\
\hline he1046-1513 & $10^{h}$ & $47^{m}$ & $00.0^{s}$ & $-15^{\circ}$ & $13^{\prime}$ & $22^{\prime \prime}$ & $24 / 04 / 95$ & $60^{m}$ & $2^{\prime \prime} .5$ & $90^{\circ}$ \\
\hline he1153-1609 & 11 & 53 & 00.6 & -16 & 09 & 08 & $23 / 04 / 95$ & $45^{m}$ & $2^{\prime \prime} .5$ & $90^{\circ}$ \\
\hline he1155-0953 & 11 & 55 & 11.5 & -09 & 53 & 45 & $25 / 04 / 95$ & $45^{m}$ & $2^{\prime \prime} .5$ & $90^{\circ}$ \\
\hline he1155-1311 & 11 & 55 & 50.8 & -13 & 11 & 37 & $25 / 04 / 95$ & $60^{m}$ & $2^{\prime \prime} .5$ & $90^{\circ}$ \\
\hline he1203-2644 & 12 & 03 & 25.0 & -26 & 44 & 11 & $25 / 04 / 95$ & $30^{m}$ & $2^{\prime \prime} .5$ & $90^{\circ}$ \\
\hline he1233-1047 & 12 & 33 & 04.1 & -10 & 47 & 33 & $25 / 04 / 95$ & $60^{m}$ & $2^{\prime \prime} .5$ & $45^{\circ}$ \\
\hline he1247-1115 & 12 & 47 & 01.7 & -11 & 15 & 19 & $23 / 04 / 95$ & $45^{m}$ & $2^{\prime \prime} .5$ & $90^{\circ}$ \\
\hline he1256-1458 & 12 & 56 & 54.7 & -14 & 58 & 11 & $23 / 04 / 95$ & $30^{m}$ & $2^{\prime \prime} .5$ & $90^{\circ}$ \\
\hline he1300-0833 & 13 & 00 & 08.0 & -08 & 33 & 48 & $23 / 04 / 95$ & $45^{m}$ & $2^{\prime \prime} .5$ & $90^{\circ}$ \\
\hline he1320-2724 & 13 & 20 & 59.6 & -27 & 24 & 03 & $24 / 04 / 95$ & $45^{m}$ & $2^{\prime \prime} .5$ & $90^{\circ}$ \\
\hline he1328-2511 & 13 & 28 & 30.6 & -25 & 11 & 55 & $23 / 04 / 95$ & $45^{m}$ & $2 " .5$ & $90^{\circ}$ \\
\hline
\end{tabular}

The observations were carried out at the $1.52 \mathrm{~m}$ telescope at ESO, and we had three nights out of four of excellent weather with a mean seeing of 0.7 arcsec. 

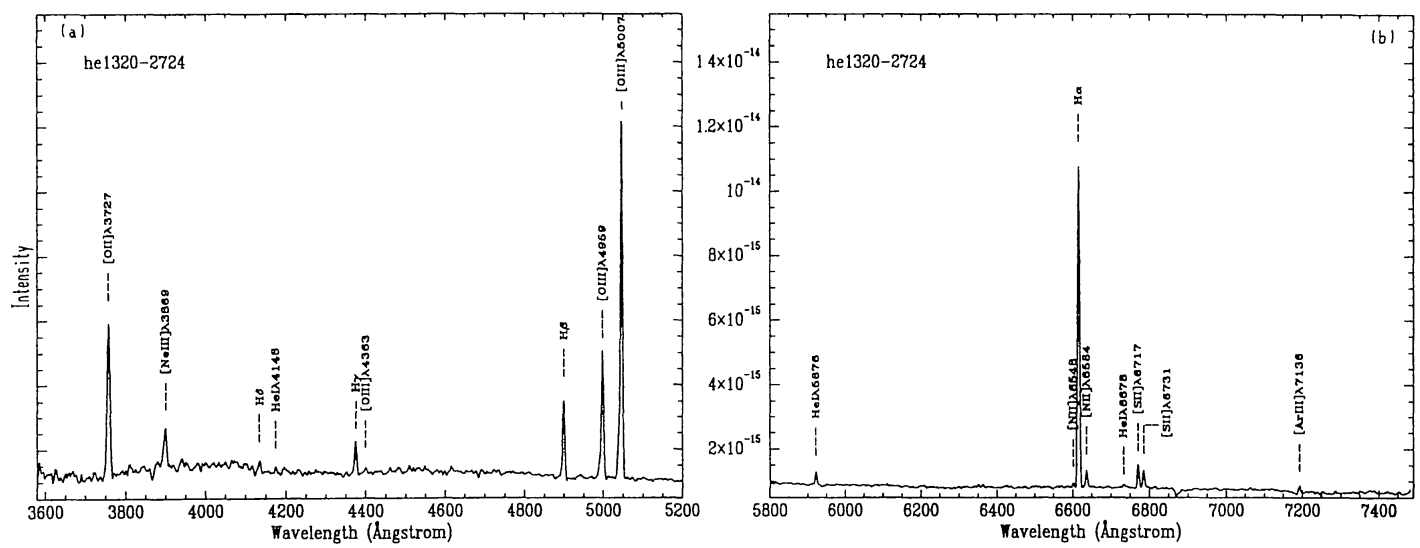

Fig. 2. Blue (a) and red (b) part of the spectrum of he1320-2724. Several emission lines, typical of HII region spectra, are shown.

3.

\section{Data reduction}

Our spectra were bias corrected, flatfielded, wavelength calibrated with He-Ar lamps and flux calibrated with spectrophotometric standard stars. Then fluxes were corrected for atmospheric extinction and galactic extinction using $E(B-V)=A(B) / 4$ and then $A(V)=3.1 E(B-V)$. Subsequently we obtained the internal extinction coefficient and the fluxes were finally corrected with this quantity.

In Fig. 2 the blue (a) and red (b) parts of the spectrum of he1320-2724 are shown.

In some cases it is possible to outline the contribution of the underlying galaxy, as can be seen in Fig. [down], in which some absorption features of the Balmer series (most probably produced by young A stars) are clearly present in the spectrum of knot IV (Fig. 3a [up]).
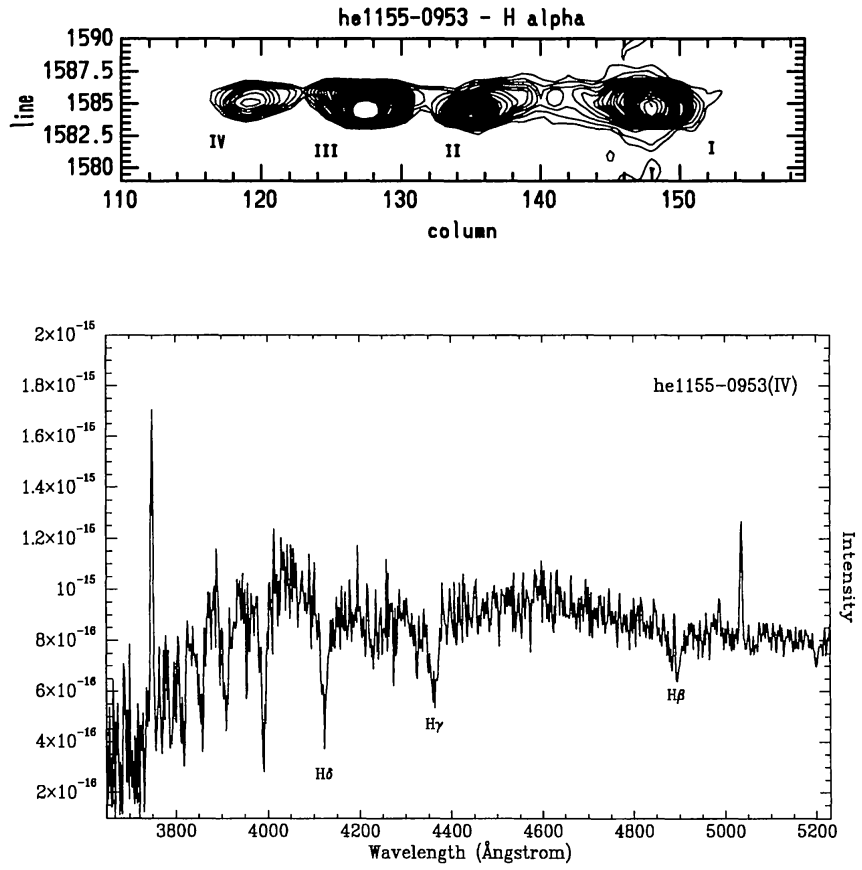

Fig. 3. [up] H $\alpha$ contour map of he1155-0953. [down] Knot IV of he1155-0953: Balmer absorption features of the underlying galaxy.

We derived the electronic densities from the [SII] doublet, the temperatures from the ratio between the nebular and auroral lines of [OIII], and then the abundances of oxygen relative to hydrogen using the formulas derived by Pagel et al. (1992) from the models of Stašinska (1990). 
For those spectra in which the [OIII] $\lambda 4363$ line was not detected, we calculated some models using the photoionization code CLOUDY (Ferland 1991), in the attempt to reproduce the observed spectrum and giving to the flux of the $\lambda 4363$ the value obtained measuring the noise at its position. For these objects calculations are in progress, but the first models seem to give densities slightly higher than those found in the other objects and metal abundances of the order of one tenth of the solar value.

In Table 2 we present deriveded metal abundances for the first group of objects, those with the [OIII] $\lambda 4363$ line clearly visible in their spectra. For comparison,purposes we remember that the solar value is $12+\log (0 / \mathrm{H})=8.92$ (corresponding to $\mathrm{Z}=0.02$ ).

Table 2. Measured electron densities, [OIII] temperatures and metallicities.

\begin{tabular}{|l|r|r|c|c|}
\hline \multicolumn{1}{|c|}{ Object } & $\begin{array}{c}\mathbf{n}_{e} \\
\left(\mathrm{~cm}^{-3}\right)\end{array}$ & $\begin{array}{c}\mathrm{T}_{e}(\mathrm{OIII}) \\
\left({ }^{\circ} \mathrm{K}\right)\end{array}$ & $12+\log (\mathrm{O} / \mathrm{H})$ & $\mathrm{Z}^{a}$ \\
\hline he1153-1609 & 298 & 25000 & 7.58 & $9.9 \mathrm{e}-4$ \\
he1155-1311 & 114 & 15400 & 7.88 & $2.0 \mathrm{e}-3$ \\
he1233-1047(I) & 315 & 10000 & 8.49 & $8.1 \mathrm{e}-3$ \\
he1247-1115 & 161 & 12000 & 8.15 & $3.8 \mathrm{e}-3$ \\
he1256-1458 & 106 & 9000 & 8.50 & $8.3 \mathrm{e}-3$ \\
he1320-2724 & 90 & 12500 & 8.23 & $4.5 \mathrm{e}-3$ \\
he1328-2511 & 25 & 13500 & 8.12 & $3.5 \mathrm{e}-3$ \\
\hline
\end{tabular}

${ }^{a} \log \mathrm{Z}=1.42+\log (\mathrm{O} / \mathrm{H})$

\section{The presence of broad lines}

The spectra of some objects of the sample show evidence of a broadening of the emission features. In Fig. [left] is represented the $\mathrm{H} \beta$ (broadened) profile (solid line), superimposed to the gaussian profile derived from $\mathrm{H} \alpha$. The FWHM of the gaussian is roughly coincident with that of the instrumental profile, so the effect seems to be real. Analogous effects are reported by Izotov et al. (1996).
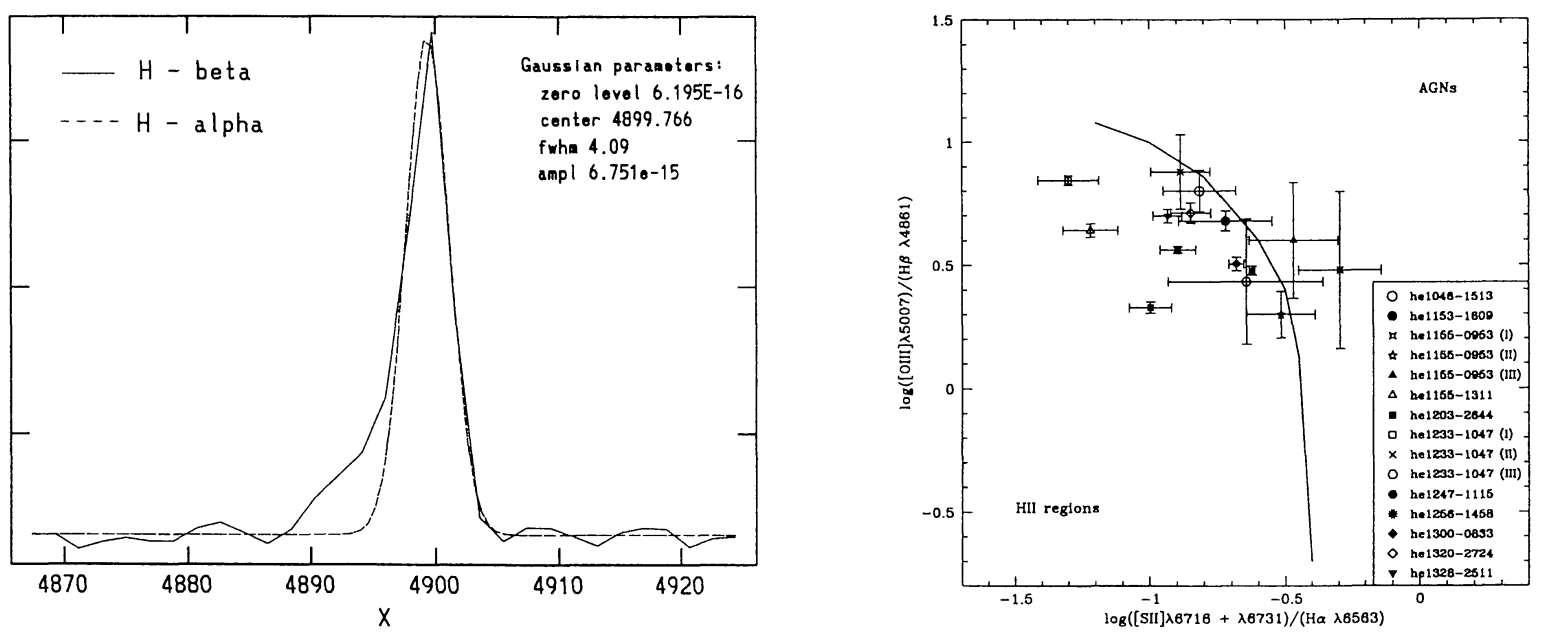

Fig. 4. [left] $\mathrm{H} \beta$ broadened profile (solid line) superimposed to $\mathrm{H} \alpha$ gaussian profile (dashed line) for he1328-2511; [right] Diagnostic diagram adapted from Baldwin et al. (1981). Some BCGs of our sample show anomalous emission line ratios.

BCGs are sites of enhanced star formation, in bursting episodes which last $\sim 10^{7}$ years. During these bursts, $10^{2}-10^{5} \mathrm{O}$ stars can be formed (Kunth \& Sargent 1983). Due to the presence of these massive and short-living stars, the dynamic of insterstellar medium (ISM) of BCGs is strongly influenced by processes such as intense stellar winds and supernova explosions. These mechanisms would transfer mechanical energy in the ISM, giving rise to bubbles of hot material and, in some cases, to shocks.

In literature are reported about 40 BCGs with evidence of broad emission features characteristic of WR stars. But WR emission lines are not observed in galaxies with oxygen abundances equal to or lower than $1 / 10$ solar, in 
agreement with predictions of evolutionary models of massive stars (Maeder 1990); this can account for the lack of emission features such as HeII $\lambda 4686$ in our spectra.

Several shock models appeared in the last few years to explain anomalous line ratios observed in some LINERS and in Seyfert's NLRs (see, f.e., Taylor et al. 1992; Dopita \& Sutherland 1995). Phenomena like shocks seem to be the natural explanation for the shape of observed emission line profiles and for the "temperature problem" which arises from the very high ratios of the forbidden oxygen lines relative to $\mathrm{H} \beta$ (Morse et al. 1996). In addition [OII] $\lambda 4363 / \mathrm{H} \beta$ ratios up to 14 are observed in our galaxies. These ratios are to high to be explained by simple photoionization

Diagnostic diagrams (from Baldwin et al. 1981), such as this in Fig. 4[right], give some insight that ionization mechanisms other than photoionization by thermal sources could be present inside some BCGs.

\section{Short summary}

Enhanced star formation is the principal explanation for BCGs properties. In fact, the spectra of our objects mostly resemble those of HII regions, with line ratios consistent with excitation by thermal sources. Metallicities are around $1 / 10$ of the solar value. So these objects are good candidates for the determination of the primordial helium content.

Some objects show line ratios non completely compatible with those expected by "pure" HII regions: this fact, combined with the presence of broadened emission features make clear that mechanisms, such as shocks generated in the turbulent ISM of these small galaxies, should be partly responsible of the observed line ratios.

\section{References}

Baldwin, J. A., Phillips, M. M., Terlevich, R. 1981, Pub. Ast. Soc. Pac. 93,5.

Campbell, A. 1992, Astrophys. J. 401, 157.

Campbell, A., Terlevich, R., Melnick, J. 1986, Mon. Not. R. Astron. Soc. 223, 811.

Dopita, M. A. 1995, Astrophys. J. 455, 468.

Ferland, G. J. 1991, Hazy: a brief introduction to CLOUDY 80.6, OSU Astronomy Department - Internal Report 91-01.

Izotov, Y.I., Thuna, T.X. Lipovetski, V. A. 1994, Astrophys. J. 435, 647.

Izotov, Y.I., Dyak, A. B., Chaffee, F. H., Foltz, C. B., Kniazev, A. Y., Lipovetski, V. A. 1996, Astrophys. J. $458,524$.

Kunth, D., Sargent, W. L. W. 1983 , Astrophys. J. 273, 81

Maeder, A. 1990, Astron. Astrophys. Suppl. Ser. 84, 139.

Morse, J. A., Raymond, J. C., Wilson, A.S. 1996, Pub. Ast. Soc. Pac. 108, 426.

Pagel, B. E. J., Simonson, E. A., Terlevich, R. J., Edmunds, M. G. 1992, Mon. Not. R. Astron. Soc. $255,325$.

Sargent, W. L. W., Searle, L. Astrophys. J. 162, L155.

Stašinska, G. 1990, Astron. Astrophys. Suppl. Ser. 83, 501.

Taylor, D., Dyson, J.E., Axon, D. J. 1992, Mon. Not. R. Astron. Soc. 255, 351.

Addresses of the authors:

A.A.M. Esposito, P. Rafanelli

Department of Astronomy - University of Padova

Vicolo dell'Osservatorio, 5

35122 Padova

Italy

V.A. Lipovetski

Special Astrophysical Observatory

Nizhny Arkhyz

357147 Russia 